\title{
Single Nucleotide Polymorphism in the Aetiology of Caries: Systematic Literature Review
}

\author{
Paula Piekoszewska-Ziętek Anna Turska-Szybka Dorota Olczak-Kowalczyk
}

Department of Paediatric Dentistry, Medical University of Warsaw, Warsaw, Poland

\section{Keywords}

Amelogenin $\cdot$ AMELX $\cdot$ Carbonic anhydrase $6 \cdot C A 6 \cdot$ Caries aetiology $\cdot$ Dental diseases $\cdot$ Genetics . Single nucleotide polymorphism

\begin{abstract}
Recent progress in the field of molecular biology and techniques of DNA sequence analysis allowed determining the meaning of hereditary factors of many common human diseases. Studies of genetic mechanisms in the aetiology of caries encompass, primarily, 4 main groups of genes responsible for (1) the development of enamel, (2) formation and composition of saliva, (3) immunological responses, and (4) carbohydrate metabolism. The aim of this study was to present current knowledge about the influence of single nucleotide polymorphism (SNP) genetic variants on the occurrence of dental caries. PubMed/Medline, Embase, and Cochrane Library databases were searched for papers on the influence of genetic factors connected with SNP on the occurrence of dental caries in children, teenagers, and adults. Thirty original papers written in English were included in this review. Study groups ranged from 30 to 13,000 subjects. SNPs were observed in 30 genes. Results of the majority of studies confirm the participation of hereditary factors in the aetiology of
\end{abstract}

\section{KARGER}

(C) 2017 S. Karger AG, Basel

E-Mail karger@karger.com

www.karger.com/cre caries. Three genes, AMELX, AQP5, and ESRRB, have the most promising evidence based on multiple replications and data, supporting a role of these genes in caries. The review of the literature proves that SNP is linked with the aetiology of dental caries.

(c) 2017 S. Karger AG, Basel

Dental caries is a complex, multifactorial illness which still appears to be one of the most common among human beings, in spite of the application of various preventive methods [Petersen, 2003]. Genetic and environmental factors, which mutually influence one another, take part in the development of caries. Their identification and knowledge of the mechanisms of influence have important meaning in the full understanding of the essence of carious disease [Wang et al., 2012b; Opal et al., 2015]. In the past century scientists became widely interested in the effect of hereditary factors on the risk of caries development. Examinations in order to explain the potential impact of genetics on caries risk at various organisms were conducted. The development of molecular biology and techniques of DNA sequence analysis initiated more advanced and reliable studies, proving the role of hereditary features in the occurrence of dental caries [Shuler, 2001;

Anna Turska-Szybka

St. Miodowa 18

PL-00-246 Warsaw (Poland)

E-Mail aturskaszybka@orange.pl 
Vieira, 2012]. There has also been considerable progress in the mapping of the human genome, which presents the position of particular genes on the chromosome. Therefore, the identification of genes contributing to increased caries susceptibility became possible [Werneck et al., 2010]. Studies of genetic mechanisms in the aetiology of caries encompass, primarily, 4 main groups of genes responsible for (1) development of the enamel, (2) formation and composition of saliva, (3) immunological response, and (4) carbohydrate metabolism [Shuler, 2001; Darshana et al., 2014]. Genome-wide scan studies conducted by Vieira et al. [2008] revealed the association of loci 5q13.3, 14q11.2, and Xq27.1 with low caries vulnerability, which was confirmed later by Shimizu et al. [2013] and Küchler et al. [2014]. On the other hand, loci 13q31.1 and 14q24.3 were connected with high caries susceptibility [Vieira et al., 2008]. Other genome-wide association studies (GWAS) suggested some loci within or near genes RPS6KA2 (chromosome 6), PTK2B (chromosome 8), RHOU (chromosome 1), FZD1 (chromosome 7), and ADMTS3 and TLR2 (chromosome 4), as well as ISL1 (chromosome 5), ACTN2 (chromosome 1), MTR (chromosome 1), and AJAP1 (chromosome 1), which can probably participate in caries development in permanent or primary dentition [Shaffer et al., 2011; Wang et al., 2012a; Shaffer et al., 2013]. Recently published GWAS by Morrison et al. [2016] conducted in a Hispanic population suggested associations in genes lacking known or plausible roles in dental caries, for example, the NAMPT gene (which is involved in many biological processes including periodontal healing) and the BMP7 gene (involved in dentin development) in the carious process. Genetic research studies concerning single nucleotide polymorphism (SNP) are positively more popular, as SNP is the most common form of human genome variability [Yang et al., 2014; Jiang et al., 2016]. The aim of this literature review was to present current knowledge about the influence of SNP genetic variants on the occurrence of dental caries.

\section{Materials and Methods}

PubMed/Medline, Embase, and Cochrane Library databases were searched for candidate gene studies on the influence of SNP genetic variants on the occurrence of dental caries using the following protocol: "dental caries susceptibility/genetics" (Medical Subject Headings, MeSH). Additionally, subsequent key words were used: "caries genetics," "caries single nucleotide polymorphism," "caries SNP," "caries genetic aetiology," and "dental caries genes." The search was complemented manually by references of the most recent pertinent reviews and original articles. The titles of the papers were verified, duplicates were excluded, and significant abstracts and, subsequently, papers were chosen and downloaded in full text. Inclusion criteria were up-to-date original papers - candidate gene studies concerning children, teenagers, or adults in which SNPs were related to genes responsible for (1) development of the tooth, (2) formation and composition of saliva, (3) host immunological response, and (4) carbohydrate metabolism. Exclusion criteria were case reports, studies on animals, and genetic studies on aspects other than SNPs (e.g., microsatellite markers, biochemical markers). The quality of the original studies was assessed using the Newcastle-Ottawa Scale (NOS).

\section{Results}

Out of 2,551 obtained records, 30 papers qualified for the review of the literature. The schema of the qualification process is presented by a PRISMA (Preferred Reporting Items for Systematic Reviews and Meta-Analyses) flow diagram (Fig. 1).

Out of the 30 qualified papers, 21 studies reported statistically significant associations between genes associated with caries and SNPs. Study groups range from 30 to nearly 13,000 subjects. SNP was observed in 30 genes. The issue of the development of the tooth was raised in $17 \mathrm{pa}-$ pers, the formation and composition of saliva in 7 , host immunological response in 7, and carbohydrate metabolism in 5. Some papers focused on SNP in 1 gene, others on SNPs in several genes. In the majority of the quoted researches caries was evaluated using the DMFT/DMFS index for permanent dentition and the $\mathrm{dmft} / \mathrm{dmfs}$ index for primary dentition, excepting Duverger et al. [2014] (caries presence/absence), Fine et al. [2013] (X-ray evaluation), and Fushan et al. [2009] (no caries evaluation). The age range of the patients was from 1 to 84 years (9 studies considered children, 9 adults, and 11 both children and adults). The results show the studied population (homogeneous vs. heterogeneous), the selected statistical approaches (univariate vs. multivariate analyses), and haplotype combination.

An overview of the study results is described in Tables $1-4$. Table 1 reports results of studies concerning genes taking part in tooth formation. Results of studies regarding genes influencing the composition and functions of saliva are introduced in Table 2 . Results of studies related to genes influencing the immune response are shown in Table 3. Table 4 reports results of studies concerning genes influencing carbohydrate metabolism. The quality of the included studies, assessed using the NOS, is shown in Table 5. The highest quality studies were awarded up to 8 stars ( 9 out of 30 studies). 


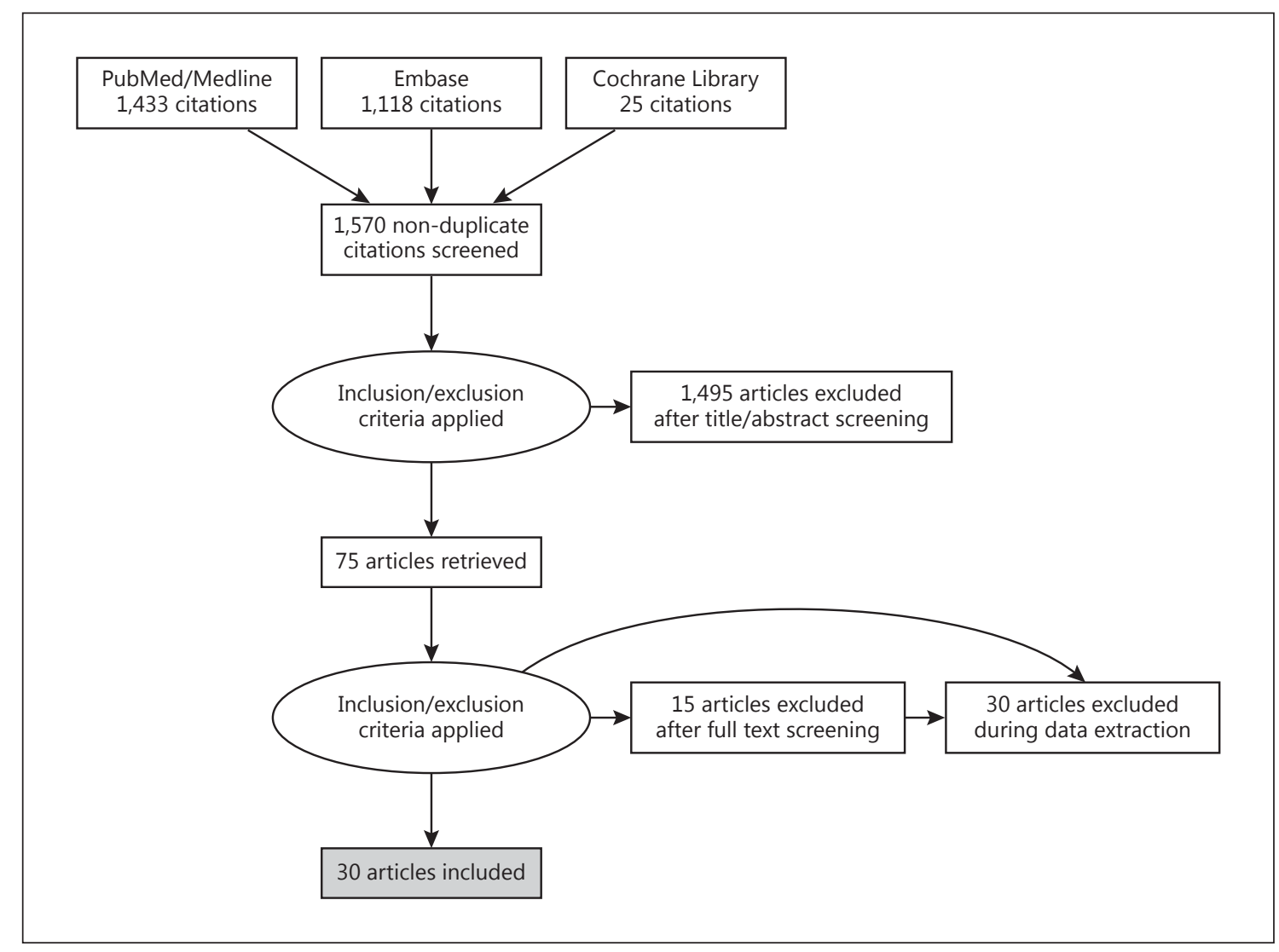

Fig. 1. PRISMA flow diagram.

\section{Discussion}

Evidence on the influence of genetic factors on the occurrence of dental caries generally comes from research studies concerning 4 main groups of genes taking part in the development of the enamel, formation and composition of saliva, immunological response, and carbohydrate metabolism. However, recent studies revealed that they can also come from other genes that previously were not expected to modulate the carious disease.

Authors of some studies included in the review observed the SNP phenomenon in genes involved in amelogenesis [Slayton et al., 2005; Deeley et al., 2008; Patir et al., 2008, 2014; Kang et al., 2011; Olszowski et al., 2012; Shimizu et al., 2012; Tannure et al., 2012a, b; Wang et al., 2012b; Gasse et al., 2013; Chaussain et al., 2014; Duverger et al. 2014; Abbasoğlu et al., 2015; Anjomshoaa et al., 2015; Shaffer et al., 2015; Yildiz et al., 2016]. Amelogenesis is a genetically controlled process of enamel development. Two main groups of proteins take part in the process: amelogenins (encoded by AMELX and AMELY

Gene Polymorphism in the Aetiology of Caries genes) and non-amelogenins - enamelin (encoded by the ENAM gene), ameloblastin (encoded by the $A M B L$ gene), and tuftelin (encoded by TUFT1 or TFIP11 genes). There are also other proteins which can assist in amelogenesis, like kallikreins (e.g., kallikrein 4 encoded by the KLK4 gene) or metalloproteinases (e.g., metalloproteinase 13, encoded by $M M P 13$, and metalloproteinase 20, encoded by MMP20) [Werneck et al., 2010; Opal et al., 2015]. The results of the quoted research studies indicate that different gene variants, connected with SNP, can be linked to higher as well as lower caries vulnerability. Patir et al. [2008] proved that increased caries susceptibility is connected with allele T of tuftelin (rs3790506) and allele C of amelogenin (rs17878486). The authors emphasize that the connection of tuftelin's SNP with dental caries revealed in the study does not depend on simultaneous analysis of Streptococcus mutans infection. Such reliance was observed earlier by Slayton et al. [2005]. On the other hand, a possible interaction of $S$. mutans infection with enamelin variants was noticed. Abbasoğlu et al. [2015] remarked the relation of some SNPs (kallikrein 4 - 
Table 1. Overview of studies concerning genes taking part in tooth formation

\begin{tabular}{|c|c|c|c|c|}
\hline Authors, year & $\begin{array}{l}\text { Study population } \\
\text { (HO/HE), } n, \\
\text { age, years }\end{array}$ & $\begin{array}{l}\text { Examples of examined } \\
\text { genes: SNP/MAF }\end{array}$ & Results & $\begin{array}{l}\text { Statistical } \\
\text { approaches }\end{array}$ \\
\hline $\begin{array}{l}\text { Morrison et al., } \\
2016\end{array}$ & $\begin{array}{l}\mathrm{HO} \\
12,803 \\
18-74\end{array}$ & $\begin{array}{l}\text { BMP7: } \\
\text { rs72626594/0.01(A) }\end{array}$ & Significant influence on caries risk & $\begin{array}{l}\text { MA } \\
p=3 \times 10^{-8}\end{array}$ \\
\hline $\begin{array}{l}\text { Yildiz et al., } \\
2016\end{array}$ & $\begin{array}{l}\mathrm{HO} \\
154 \\
20-60\end{array}$ & $\begin{array}{l}\text { AMELX: } \\
\text { rs6639060/0.46(T) }\end{array}$ & No influence on caries occurrence & $\begin{array}{l}\text { MA } \\
\text { Not significant } \\
p>0.05\end{array}$ \\
\hline \multirow[t]{2}{*}{$\begin{array}{l}\text { Abbasoğlu et al., } \\
2015\end{array}$} & $\begin{array}{l}\mathrm{HO} \\
259 \\
4.6 \pm 0.61\end{array}$ & $\begin{array}{l}\text { ENAM: } \\
\text { rs1264848/0.11(C) } \\
\text { KLK4: } \\
\text { rs198968/0.32(A) } \\
\text { TUFT1: } \\
\text { rs3790506/0.25(A) }\end{array}$ & GG genotypes protective for ECC & $\begin{array}{l}\text { MA } \\
p=0.032 \\
\text { MA } \\
p=0.040 \\
\mathrm{UA}, \mathrm{MA} \\
p=0.014\end{array}$ \\
\hline & & $\begin{array}{l}\text { ALOX15: } \\
\text { rs7217186/0.47(T) }\end{array}$ & TT genotype is a risk factor for ECC & $\begin{array}{l}\text { MA } \\
p=0.050\end{array}$ \\
\hline $\begin{array}{l}\text { Anjomshoaa et al., } \\
2015\end{array}$ & $\begin{array}{l}\mathrm{HE} \\
1,383 \\
2-84\end{array}$ & $\begin{array}{l}A Q P 5: \\
\text { rs3759129/0.08(C) } \\
\text { rs467323/0.35(T) } \\
\text { rs1996315/0.43(A) } \\
\text { rs10875989/0.48(C) }\end{array}$ & $\begin{array}{l}\text { Significant influence on caries } \\
\text { experience } \\
\text { Haplotype rs461872-rs1996315 } \\
\text { (dominant model) } \\
\text { Haplotype rs3759129-rs1996315 } \\
\text { (recessive model) }\end{array}$ & $\begin{array}{l}\text { MA } \\
p=0.03 \\
p=0.01\end{array}$ \\
\hline \multirow[t]{2}{*}{$\begin{array}{l}\text { Shaffer et al., } \\
2015\end{array}$} & $\begin{array}{l}\mathrm{HE} \\
3,600 \\
3-12 \\
18-79\end{array}$ & $\begin{array}{l}\text { TUFT1: } \\
\text { rs2337359/0.47(C) }\end{array}$ & $\begin{array}{l}\text { Significant relation with caries in } \\
\text { adults; no SNPs showed association } \\
\text { in both children and adults }\end{array}$ & $\begin{array}{l}\text { MA } \\
p<0.002\end{array}$ \\
\hline & & $\begin{array}{l}\text { TUFT1: } \\
\text { rs2337359/0.47(C) } \\
\text { AMBN: } \\
\text { rs7439186/0.12(T) }\end{array}$ & $\begin{array}{l}\text { Relation of SNP variant with type of } \\
\text { fluorine exposure }\end{array}$ & $\begin{array}{l}\text { MA } \\
p<0.05\end{array}$ \\
\hline $\begin{array}{l}\text { Chaussain et al., } \\
2014\end{array}$ & $\begin{array}{l}\mathrm{HO} \\
358 \\
7.6 \pm 2.9 \\
22.0 \pm 1.8\end{array}$ & $\begin{array}{l}\text { ENAM: } \\
\text { rs2609428/0.04(C) } \\
\text { rs7671281/0.12(C) } \\
\text { rs3796704/0.10(A) }\end{array}$ & $\begin{array}{l}\text { rs2609428 associated with caries } \\
(\mathrm{OR}, 3.89 ; 95 \% \mathrm{CI}, 1.47-10.33) \\
\text { Haplotype rs7671281-rs3796704 } \\
\text { associated with caries } \\
(\mathrm{OR}, 2.66 ; 95 \% \mathrm{CI}, 0.99-7.20)\end{array}$ & $\begin{array}{l}\text { UA } \\
p<0.05 \\
\text { MA } \\
p<0.05\end{array}$ \\
\hline $\begin{array}{l}\text { Duverger et al., } \\
2014\end{array}$ & $\begin{array}{l}\mathrm{HO} \\
1,092 \\
6-12 \\
25-50\end{array}$ & $\begin{array}{l}\text { KRT75: } \\
\text { rs2232387/0.14(T) }\end{array}$ & Allele A increases caries susceptibility & $\begin{array}{l}\mathrm{UA} \\
p=0.0175\end{array}$ \\
\hline $\begin{array}{l}\text { Gasse et al., } \\
2013\end{array}$ & $\begin{array}{l}\mathrm{HE} \\
358 \\
2-17\end{array}$ & $\begin{array}{l}\text { AMELX: } \\
\text { rs184371797/0.001(C) } \\
\text { rs946252/0.30(T) } \\
\text { rs200163085/0.01(G) }\end{array}$ & No influence on caries occurrence & $\begin{array}{l}\text { UA, MA } \\
\text { Not significant } \\
p>0.05\end{array}$ \\
\hline $\begin{array}{l}\text { Olszowski et al., } \\
2012\end{array}$ & $\begin{array}{l}\mathrm{HO} \\
179 \\
5,13\end{array}$ & $\begin{array}{l}\text { AMELX: } \\
\text { rs2106416/0.16(T) }\end{array}$ & No influence on caries occurrence & $\begin{array}{l}\text { UA } \\
\text { Not significant } \\
p>0.05\end{array}$ \\
\hline
\end{tabular}


Table 1 (continued)

\begin{tabular}{|c|c|c|c|c|}
\hline Authors, year & $\begin{array}{l}\text { Study population } \\
\text { (HO/HE), } n, \\
\text { age, years }\end{array}$ & $\begin{array}{l}\text { Examples of examined } \\
\text { genes: SNP/MAF }\end{array}$ & Results & $\begin{array}{l}\text { Statistical } \\
\text { approaches }\end{array}$ \\
\hline $\begin{array}{l}\text { Shimizu et al., } \\
2012\end{array}$ & $\begin{array}{l}\mathrm{HE} \\
1,831 \\
1-82\end{array}$ & $\begin{array}{l}\text { AMELX: } \\
\text { rs946252/0.31(T) } \\
\text { TUFT1: } \\
\text { rs4970957/0.22(G) } \\
\text { AMBN: } \\
\text { rs4694075/0.48(C) } \\
\text { ENAM: } \\
\text { rs12640848/0.33(G) }\end{array}$ & $\begin{array}{l}\text { Allele T (rs946252) and allele C } \\
\text { (rs4694075) increase caries } \\
\text { susceptibility }\end{array}$ & $\begin{array}{l}\text { MA } \\
p=0.01 \\
p=0.007\end{array}$ \\
\hline $\begin{array}{l}\text { Tannure et al., } \\
\text { 2012b }\end{array}$ & $\begin{array}{l}\mathrm{HE} \\
388 \\
9.03 \pm 2.75\end{array}$ & $\begin{array}{l}\text { MMP20: } \\
\text { rs1784418/0.42(T) }\end{array}$ & Allele T may increase caries risk & $\begin{array}{l}\text { MA } \\
\text { Not significant } \\
p>0.05\end{array}$ \\
\hline $\begin{array}{l}\text { Tannure et al., } \\
\text { 2012a }\end{array}$ & $\begin{array}{l}\mathrm{HE} \\
505 \\
3-21\end{array}$ & $\begin{array}{l}\text { MMP13: } \\
\text { rs2252070/0.36(C) }\end{array}$ & GG genotype increases caries risk & $\begin{array}{l}\text { MA } \\
p=0.004 \\
(\mathrm{OR}, 0.538 ; 95 \% \\
\text { CI, } 0.313-0.926)\end{array}$ \\
\hline $\begin{array}{l}\text { Wang et al., } \\
2012 \text { b }\end{array}$ & $\begin{array}{l}\mathrm{HE} \\
333 \\
4-7\end{array}$ & $\begin{array}{l}D S P P: \\
\text { rs2615487/0.32(T) } \\
\text { KLK4: } \\
\text { rs2235091/0.36(G) }\end{array}$ & $\begin{array}{l}\text { Allele A (rs2235091) and allele T } \\
\text { increase caries resistance }\end{array}$ & $\begin{array}{l}\text { MA } \\
p<0.05\end{array}$ \\
\hline $\begin{array}{l}\text { Kang et al., } \\
2011\end{array}$ & $\begin{array}{l}\mathrm{HE} \\
120 \\
22.7 \pm 7.8\end{array}$ & $\begin{array}{l}A M E L X: \\
\text { rs17878486/0.08(C) } \\
\text { rs5933871/0.27(C) } \\
\text { rs5934997/0.27(C) }\end{array}$ & $\begin{array}{l}\text { TT genotypes (rs5933871; rs5934997) } \\
\text { increase caries susceptibility }\end{array}$ & $\begin{array}{l}\text { MA } \\
p=0.001 \\
p=0.000\end{array}$ \\
\hline $\begin{array}{l}\text { Deeley et al., } \\
2008\end{array}$ & $\begin{array}{l}\mathrm{HO} \\
110 \\
7-14\end{array}$ & $\begin{array}{l}A M B N: \\
\text { hCV496502/0.22(T) } \\
\text { AMELX: } \\
\text { hCV2190967/0.06(T) }\end{array}$ & $\begin{array}{l}\text { Variants of amelogenin gene are } \\
\text { connected with higher caries } \\
\text { susceptibility }\end{array}$ & $\begin{array}{l}\mathrm{UA} \\
p=0.0000001\end{array}$ \\
\hline $\begin{array}{l}\text { Patir et al., } \\
2008\end{array}$ & $\begin{array}{l}\text { HO } \\
173 \\
4.82 \pm 0.81\end{array}$ & $\begin{array}{l}A M B N: \\
\text { rs34538475/0.17(T) } \\
\text { AMELX: } \\
\text { rs178784860.08(C) } \\
\text { TUFT1: } \\
\text { rs3796704/0.14(A) } \\
\text { ENAM: } \\
\text { rs3790506/0.25(A) }\end{array}$ & $\begin{array}{l}\text { Allele T (rs3790506) and allele C } \\
\text { (rs17878486) increase caries } \\
\text { susceptibility }\end{array}$ & $\begin{array}{l}\text { MA } \\
p=0.05 \\
p=0.01\end{array}$ \\
\hline $\begin{array}{l}\text { Slayton et al., } \\
2005\end{array}$ & $\begin{array}{l}\mathrm{HE} \\
470 \\
3-5\end{array}$ & $\begin{array}{l}\text { TUFT1: } \\
\text { CTTCTCAAGGT/CTG- } \\
\text { TAGGAAGA /0.26(A) }\end{array}$ & $\begin{array}{l}\text { Variants of tuftelin gene connected with } \\
\text { high } S \text {. mutans quantity may predispose } \\
\text { to caries }\end{array}$ & $\begin{array}{l}\text { MA } \\
\text { Not significant } \\
p>0.05\end{array}$ \\
\hline
\end{tabular}

HO, homogeneous; HE, heterogeneous; ECC, early childhood caries; UA, univariate analysis; MA, multivariate analysis; MAF, minor allele frequency.

rs198968, enamelin - rs1264848, lactoferrin - rs4547741, and tuftelin - rs3790506) with decreased caries susceptibility. Particular genotypes in the examined patients were determined as protective against early childhood caries. Similar results were achieved by Wang et al. [2012b], who created a comparison of alleles, which predispose to a decreased risk of smooth surfaces or pit/fissure caries, after they analysed polymorphic variants of genes (KLK4 rs2235091, DSPP - rs2615487, and AQP5 - rs1996315). Different results were obtained by Olszowski et al. [2012] 
Table 2. Overview of studies concerning genes influencing composition and functions of saliva

\begin{tabular}{|c|c|c|c|c|}
\hline Authors, year & $\begin{array}{l}\text { Study population } \\
\text { (HO/HE), } n, \\
\text { age, years }\end{array}$ & $\begin{array}{l}\text { Examples of examined } \\
\text { genes: SNP/MAF }\end{array}$ & Results & $\begin{array}{l}\text { Statistical } \\
\text { approaches }\end{array}$ \\
\hline $\begin{array}{l}\text { Yildiz et al., } \\
2016\end{array}$ & $\begin{array}{l}\mathrm{HO} \\
154 \\
20-60\end{array}$ & $\begin{array}{l}\text { CA6: } \\
\text { rs2274327/0.27(T) }\end{array}$ & $\begin{array}{l}\text { Allele } T \text { less frequent in } \\
\text { patients with high buffer } \\
\text { capacity }\end{array}$ & $\begin{array}{l}\text { MA } \\
p<0.05\end{array}$ \\
\hline $\begin{array}{l}\text { Li et al., } \\
2014\end{array}$ & $\begin{array}{l}\mathrm{HO} \\
355 \\
51.16 \pm 9.48\end{array}$ & $\begin{array}{l}\text { CA6: } \\
\text { rs2274328/0.50(A) } \\
\text { rs17032907/0.22(T) } \\
\text { rs11576766/0.35(C) } \\
\text { rs2274333/0.34(G) } \\
\text { rs10864376/0.47(C) } \\
\text { rs3765964/0.46(A) } \\
\text { rs6680186/0.40(G) }\end{array}$ & $\begin{array}{l}\text { Haplotype ACA } \\
\text { (rs17032907) can be } \\
\text { connected with higher } \\
\text { caries susceptibility }\end{array}$ & $\begin{array}{l}\text { MA } \\
\text { Not significant } \\
p>0.05\end{array}$ \\
\hline $\begin{array}{l}\text { Buczkowska-Radlińska et al., } \\
2012\end{array}$ & $\begin{array}{l}\mathrm{HO} \\
158 \\
20-21\end{array}$ & $\begin{array}{l}\text { MUC7: } \\
\text { Exon 3/0.22 }\end{array}$ & $\begin{array}{l}\text { No influence on caries } \\
\text { intensity }\end{array}$ & $\begin{array}{l}\text { UA } \\
\text { Not significant } \\
p>0.05\end{array}$ \\
\hline $\begin{array}{l}\text { Koç Öztürk et al., } \\
2012\end{array}$ & $\begin{array}{l}\mathrm{HO} \\
43 \\
45.3 \pm 13.5\end{array}$ & $\begin{array}{l}\text { CA6: } \\
\text { rs2274327/0.27(T) } \\
\text { rs2274328/0.50(A) } \\
\text { rs2274329/0.09(C) }\end{array}$ & $\begin{array}{l}\text { Positive correlation with } \\
\text { CA6 activity }\end{array}$ & $\begin{array}{l}\mathrm{UA} \\
r=0.427 \\
p<0.05\end{array}$ \\
\hline $\begin{array}{l}\text { Wang et al., } \\
2012 \text { b }\end{array}$ & $\begin{array}{l}\mathrm{HE} \\
333 \\
4-7\end{array}$ & $\begin{array}{l}A Q P: \\
\text { rs923911/0.22(A) } \\
\text { rs1996315/0.43(A) }\end{array}$ & $\begin{array}{l}\text { Allele A increases caries } \\
\text { resistance } \\
\text { GC haplotype protective } \\
\text { for caries } \\
\text { GG haplotype is risk } \\
\text { for caries }\end{array}$ & $\begin{array}{l}\text { MA } \\
p<0.05 \\
p=0.02 \\
p=0.03\end{array}$ \\
\hline $\begin{array}{l}\text { Yarat et al., } \\
2011\end{array}$ & $\begin{array}{l}\mathrm{HO} \\
44 \\
21.95 \pm 2.2\end{array}$ & $\begin{array}{l}\text { CA6: dbSNP } \\
142460367 / 0.02(\mathrm{~A}) \\
142460368 / 0.01(\mathrm{~A})\end{array}$ & $\begin{array}{l}\text { No influence on caries } \\
\text { susceptibility }\end{array}$ & $\begin{array}{l}\text { UA } \\
\text { Not significant } \\
p>0.05\end{array}$ \\
\hline $\begin{array}{l}\text { Peres et al., } \\
2010\end{array}$ & $\begin{array}{l}\mathrm{HO} \\
245 \\
7.84 \pm 0.81\end{array}$ & $\begin{array}{l}\text { CA6: } \\
\text { rs2274327/0.27(T) } \\
\text { rs2274328/0.50(A) } \\
\text { rs2274333/0.34(G) }\end{array}$ & $\begin{array}{l}\text { TT genotype (rs2274327) } \\
\text { less frequent in high buffer } \\
\text { capacity }\end{array}$ & $\begin{array}{l}\mathrm{UA} \\
p=0.023\end{array}$ \\
\hline
\end{tabular}

HO, homogeneous; HE, heterogeneous; UA, univariate analysis; MA, multivariate analysis; MAF, minor allele frequency.

and Yildiz et al. [2016], who failed to prove any connection of SNP of amelogenesis genes with dental caries vulnerability. There is a possibility that it was caused by inappropriate SNP selection (minor allele frequency in the population) or too small a number of SNPs checked for 1 gene. In contrast to authors focusing on amelogenesis, Morrison et al. [2016] noted the occurrence of SNP in genes stimulating the development of dentin (BMP7), which was not previously mentioned in the literature.

Various components of saliva influence its different functions, which contribute to maintaining oral homeo- stasis and tooth protection. Carbonic anhydrase 6 is a salivary enzyme which is assigned to regulate the buffer capacity and $\mathrm{pH}$ of saliva. Mucins and proline-rich proteins take part in bacteria cell agglutination, including cariogenic bacteria [Darshana et al., 2014; Opal et al., 2015]. Researchers examined SNP in genes encoding salivary components - CA6 (for carbonic anhydrase 6), MUC 7 (for mucin), $A Q P$ (for aquaporin), and PRH1 (for prolinerich protein 1) [Peres et al., 2010; Yarat et al., 2011; Buczkowska-Radlińska et al., 2012; Koç Öztürk et al., 2012; Wang et al., 2012b; Li et al., 2015; Yildiz et al., 2016]. 
Table 3. Overview of studies concerning genes influencing immune responses

\begin{tabular}{|c|c|c|c|c|}
\hline Authors, year & $\begin{array}{l}\text { Study population } \\
(\mathrm{HO} / \mathrm{HE}), n, \\
\text { age, years }\end{array}$ & $\begin{array}{l}\text { Examples of examined } \\
\text { genes: SNP/MAF }\end{array}$ & Results & $\begin{array}{l}\text { Statistical } \\
\text { approaches }\end{array}$ \\
\hline $\begin{array}{l}\text { Yildiz et al., } \\
2016\end{array}$ & $\begin{array}{l}\mathrm{HO} \\
154 \\
20-60\end{array}$ & $\begin{array}{l}D E F B 1: \\
\text { rs11362/0.40(T) }\end{array}$ & $\begin{array}{l}\text { Allele A more frequent in high caries } \\
\text { risk }\end{array}$ & $\begin{array}{l}\text { MA } \\
p=0.000\end{array}$ \\
\hline $\begin{array}{l}\text { Abbasoğlu et al., } \\
2015\end{array}$ & $\begin{array}{l}\mathrm{HO} \\
259 \\
4.6 \pm 0.61\end{array}$ & $\begin{array}{l}L T F: \\
\text { rs4547741/0.07(T) } \\
\text { BEFB1: } \\
\text { rs11362/0.40(T) }\end{array}$ & $\begin{array}{l}\text { CT genotype (rs4547741) is a } \\
\text { protective factor for ECC }\end{array}$ & $\begin{array}{l}\mathrm{UA}, \mathrm{MA} \\
p=0.038\end{array}$ \\
\hline $\begin{array}{l}\text { Krasone et al., } \\
2014\end{array}$ & $\begin{array}{l}\mathrm{HO} \\
69 \\
2-12\end{array}$ & $\begin{array}{l}\text { DEFB1: } \\
\text { rs11362/0.40(T) } \\
\text { rs1800972/0.14(C) }\end{array}$ & $\begin{array}{l}\text { CC genotype (rs11362) connected } \\
\text { with high caries susceptibility }\end{array}$ & $\begin{array}{l}\mathrm{UA} \\
p=0.031 \\
(\mathrm{OR}, 3.16 ; 95 \% \mathrm{CI}, \\
0.97-10.62)\end{array}$ \\
\hline $\begin{array}{l}\text { Fine et al., } \\
2013\end{array}$ & $\begin{array}{l}\mathrm{HE} \\
30 \\
34.6 \pm 9.1\end{array}$ & $\begin{array}{l}\text { LTF: } \\
\text { rs1126478/0.37(T) }\end{array}$ & $\begin{array}{l}\text { Variant LTF/K connected with high } \\
\text { lactoferrin activity against } S \text {. mutans } \\
\text { and low caries intensity }\end{array}$ & $\begin{array}{l}\mathrm{UA} \\
p=0.02 \\
(\mathrm{RR}, 3.6 ; 95 \% \mathrm{CI} \\
1.5-11.13)\end{array}$ \\
\hline $\begin{array}{l}\text { Olszowski et al., } \\
2012\end{array}$ & $\begin{array}{l}\mathrm{HO} \\
179 \\
5 \text { and } 13\end{array}$ & $\begin{array}{l}M B L 2: \\
\text { rs7096206/0.20(G) } \\
\text { rs1800450/0.12(T) } \\
M A S P 2: \\
\text { rs72550870/0.01(C) }\end{array}$ & $\begin{array}{l}\text { Allele G (rs7096206) more frequent } \\
\text { in high caries intensity }\end{array}$ & $\begin{array}{l}\text { UA } \\
\text { Not significant } \\
p>0.05\end{array}$ \\
\hline $\begin{array}{l}\text { Azevedo et al., } \\
2010\end{array}$ & $\begin{array}{l}\mathrm{HO} \\
110 \\
12\end{array}$ & $\begin{array}{l}\text { LTF: } \\
\text { rs1126478/0.37(T) }\end{array}$ & $\begin{array}{l}\text { Allele A connected with high } \\
\text { caries susceptibility }\end{array}$ & $\begin{array}{l}\mathrm{UA} \\
p=0.01 \\
(\mathrm{OR}, 0.16 ; 95 \% \mathrm{CI}, \\
0.03-0.76)\end{array}$ \\
\hline $\begin{array}{l}\text { Ozturk et al., } \\
2010\end{array}$ & $\begin{array}{l}\mathrm{HE} \\
296 \\
17-84\end{array}$ & $\begin{array}{l}\text { DEFB1: } \\
\text { rs113620.40(T) } \\
\text { rs179946/0.42(A) } \\
\text { rs1800972/0.14(C) }\end{array}$ & $\begin{array}{l}\text { Haplotype GCA connected with } \\
\text { high caries intensity } \\
\text { Haplotype ACG connected with low } \\
\text { caries intensity }\end{array}$ & $\begin{array}{l}\text { MA } \\
p=0.0023, \\
(\mathrm{OR}, 2.19 ; 95 \% \mathrm{CI}, \\
1.1-4.35) \\
p=0.0010\end{array}$ \\
\hline
\end{tabular}

HO, homogeneous; HE, heterogeneous; ECC, early childhood caries; UA, univariate analysis; MA, multivariate analysis; MAF, minor allele frequency.

The relation of the CA6 gene with the buffer capacity of saliva was observed by Yildiz et al. [2016] and Peres et al. [2010]. In both studies it concerned less frequent occurrence of the T allele or the TT genotype (rs2274327) in patients with high buffer capacity. The $\mathrm{T}$ allele was also frequently prevalent in high caries intensity; however, the differences were not statistically significant. Variant results on the same gene were obtained by Yarat et al. [2011], who linked SNP neither with the buffer capacity of saliva nor with the values of the $\mathrm{dmft}$ index (in the first group of patients $\mathrm{dmft}=0$, and in the second group $<6$ ).

Hereditary factors can affect the immune response of the organism. However, the vast majority of the re- search studies concerning this issue pertained to human leukocyte antigens, and were not SNP analyses. For this reason they were not included in the review. In this category of genes, we included studies analysing SNPs of genes encoding antimicrobial salivary peptides - LTF (encoding lactoferrin), $D E F B 1$ (encoding $\beta$-defensin 1 ), and $M B L$ and MASP2 (these are connected with mannose-binding protein produced in the liver, which participates in innate immune response) [Azevedo et al., 2010; Ozturk et al., 2010; Olszowski et al., 2012; Fine et al., 2013; Krasone et al., 2014; Abbasoğlu et al., 2015; Yildiz et al, 2016]. Azevedo et al. [2010] noticed the relation of allele A of lactoferrin (exon $2 L T F$ ) with de- 
Table 4. Overview of studies concerning genes influencing carbohydrate metabolism

\begin{tabular}{|c|c|c|c|c|}
\hline Authors, year & $\begin{array}{l}\text { Study population } \\
\text { (HO/HE), } n, \\
\text { age, years }\end{array}$ & $\begin{array}{l}\text { Examples of examined } \\
\text { genes: SNP/MAF }\end{array}$ & Results & $\begin{array}{l}\text { Statistical } \\
\text { approaches }\end{array}$ \\
\hline $\begin{array}{l}\text { Yildiz et al., } \\
2016\end{array}$ & $\begin{array}{l}\mathrm{HO} \\
154 \\
20-60\end{array}$ & $\begin{array}{l}\text { TAS2R38: } \\
\text { rs713598/0.50(C) }\end{array}$ & $\begin{array}{l}\text { GG genotype more frequent in } \\
\text { low caries intensity }\end{array}$ & $\begin{array}{l}\text { MA } \\
p=0.000\end{array}$ \\
\hline $\begin{array}{l}\text { Haznedaroğlu et al., } \\
2015\end{array}$ & $\begin{array}{l}\mathrm{HO} \\
184 \\
7-12\end{array}$ & $\begin{array}{l}\text { TAS1R2: } \\
\text { rs35874116/0.27(C) } \\
\text { rs9701796/0.20(G) } \\
\text { TAS1R3: } \\
\text { rs307355/0.24(T) }\end{array}$ & $\begin{array}{l}\text { Higher caries intensity in TT } \\
\text { homozygotes }\end{array}$ & $\begin{array}{l}\mathrm{UA}, \mathrm{MA} \\
p=0.008 \\
p=0.04\end{array}$ \\
\hline $\begin{array}{l}\text { Robino et al., } \\
2015\end{array}$ & $\begin{array}{l}\mathrm{HO} \\
647 \\
44.9 \pm 12.4\end{array}$ & $\begin{array}{l}\text { TAS1R2: } \\
\text { rs3935570/0.25(T) } \\
\text { GLUT2: } \\
\text { rs1499821/0.14(T) }\end{array}$ & $\begin{array}{l}\text { GG genotype more frequent in } \\
\text { high caries intensity }\end{array}$ & $\begin{array}{l}\text { MA } \\
p=0.0117 \\
p=0.0273\end{array}$ \\
\hline $\begin{array}{l}\text { Wendell et al., } \\
2010\end{array}$ & $\begin{array}{l}\mathrm{HO} \\
2,449 \\
1-42\end{array}$ & $\begin{array}{l}\text { TASR38: } \\
\text { rs713598/0.50(C) } \\
\text { rs1726866/0.43(A) } \\
\text { rs10246939/0.48(T) } \\
\text { TAS1R2: } \\
\text { rs4920566/0.44(A) } \\
\text { rs9701796/0.20(G) } \\
\text { GNAT3: } \\
\text { rs2074674/0.47(A) } \\
\text { rs6962693/0.27(G) }\end{array}$ & $\begin{array}{l}\text { rs } 9701796 \text { allele C connected } \\
\text { with both high and low caries risk }\end{array}$ & $\begin{array}{l}\mathrm{UA} \\
p=0.02 \\
p=0.03\end{array}$ \\
\hline $\begin{array}{l}\text { Fushan et al., } \\
2009\end{array}$ & $\begin{array}{l}\mathrm{HE} \\
144 \\
\text { No data }\end{array}$ & $\begin{array}{l}\text { TAS2R38: } \\
\text { rs307355/0.24(T) } \\
\text { rs35744813/0.28(T) }\end{array}$ & $\begin{array}{l}\text { Allele T connected with lower } \\
\text { AUC saccharose index }\end{array}$ & $\begin{array}{l}\text { UA } \\
\text { Not significant } \\
p>0.05\end{array}$ \\
\hline
\end{tabular}

HO, homogeneous; HE, heterogeneous; UA, univariate analysis; MA, multivariate analysis; MAF, minor allele frequency.

creased caries intensity, as well as with increased salivary flow, which indicates its protective activity. A connection of a variant of the LTF gene with decreased caries susceptibility was observed also by Abbasoğlu et al. [2015], who defined the CT genotype as a protective factor for early childhood caries. Interesting results were also obtained by Fine et al. [2013], who observed an $L T F$ gene variant that is connected with the activity of lactoferrin in saliva, which contributes to better antimicrobial response. It results in lower caries intensity in patients carrying this variant. On the other hand, Brancher et al. [2011] did not observe any SNP in the examined promoter region of the LTF gene in 687 twelve-year-olds of Caucasian origin.

The reception of sweet taste is stimulated by heterodimeric G proteins, encoded in TAS1R2 and TAS1R3 genes, and the bitter taste feeling is connected with the TAS2R38 gene. These influence dietary habits as they cause sensitiveness or insensitiveness to some tastes [Opal et al., 2015]. Genetically conditioned sensitiveness to bitter or sweet taste may be connected with dietary preferences (cariogenic/non-cariogenic). An example can be sensitiveness/insensitiveness to PTC (phenylthiocarbamide) or PROP (propylthiouracil). Patients who are oversensitive may feel bitter or sweet taste more intensely, therefore they need a lower concentration of a substrate and, consequently, are less susceptible to caries [Darshana et al., 2014]. SNP of genes influencing taste reception, and thus carbohydrate metabolism, is a genetic factor in the aetiology of dental caries [Fushan et al., 2009; Wendell et al., 2010; Haznedaroğlu et al., 2015; Robino et al., 2015; Yildiz et al., 2016]. Hereditary fructose intolerance can 
Table 5. The assessment of quality of the included studies using the Newcastle-Ottawa Scale

\begin{tabular}{|c|c|c|c|c|}
\hline Authors, year & Selection & Comparability & Exposure & Total \\
\hline Morrison et al., 2016 & $* * * *$ & $*$ & $* *$ & 7 \\
\hline Yildiz et al., 2016 & $* * * *$ & $*$ & $* *$ & 7 \\
\hline Abbasoğlu et al., 2015 & $* * * *$ & $* *$ & $* *$ & 8 \\
\hline Anjomshoaa et al., 2015 & $* * * *$ & $* *$ & $* *$ & 8 \\
\hline Haznedaroğlu et al., 2015 & $* *$ & $*$ & $* *$ & 5 \\
\hline Robino et al., 2015 & $* *$ & $* *$ & $* *$ & 6 \\
\hline Shaffer et al., 2015 & ** & $* *$ & $* *$ & 6 \\
\hline Chaussain al., 2014 & $* * * *$ & $* *$ & $* *$ & 8 \\
\hline Duverger et al., 2014 & $* *$ & $*$ & $* *$ & 5 \\
\hline Krasone et al., 2014 & $* * *$ & $*$ & $* *$ & 6 \\
\hline Li et al., 2014 & $* * * *$ & $*$ & $* *$ & 7 \\
\hline Fine et al., 2013 & $* *$ & $* *$ & $* *$ & 6 \\
\hline Gasse et al., 2013 & $* * * *$ & $*$ & $* *$ & 7 \\
\hline Buczkowska-Radlińska et al., 2012 & $* *$ & $*$ & $* *$ & 5 \\
\hline Koç Öztürk et al., 2012 & $* * *$ & $*$ & $* *$ & 6 \\
\hline Olszowski et al., 2012 & $* *$ & $*$ & $* *$ & 5 \\
\hline Shimizu et al., 2012 & $* * * *$ & $* *$ & $* *$ & 8 \\
\hline Tannure et al., 2012b & $* *$ & $* *$ & $* *$ & 6 \\
\hline Tannure et al., 2012a & $* *$ & $* *$ & $* *$ & 6 \\
\hline Wang et al., 2012b & $* * * *$ & $* *$ & $* *$ & 8 \\
\hline Kang et al., 2011 & $* * * *$ & $* *$ & $* *$ & 8 \\
\hline Yarat et al., 2011 & $* * *$ & $* *$ & $* *$ & 7 \\
\hline Azevedo et al., 2010 & $* * *$ & $*$ & $* *$ & 6 \\
\hline Ozturk et al., 2010 & $* * * *$ & $* *$ & $* *$ & 8 \\
\hline Peres et al., 2010 & $* * *$ & $* *$ & $* *$ & 7 \\
\hline Wendell et al., 2010 & $* * * *$ & $*$ & $* *$ & 7 \\
\hline Fushan et al., 2009 & $*$ & $*$ & $* *$ & 4 \\
\hline Deeley et al., 2008 & $* * *$ & $*$ & $* *$ & 6 \\
\hline Patir et al., 2008 & $* * * *$ & $* *$ & $* *$ & 8 \\
\hline Slayton et al., 2005 & $* * * *$ & $* *$ & $* *$ & 8 \\
\hline
\end{tabular}

lead to a variety of unpleasant symptoms, which occur in patients after its consumption. Therefore, they often intentionally avoid sweet food and, subsequently, have low caries intensity [Shuler, 2001].

Interesting research was presented by Weber et al. [2014]; however, it was not included in the review because of a different type of gene analysed. They examined the $E S R R B$ gene for the oestrogen-related receptor- $\beta$, the mutation of which can lead to hearing impairment. The authors observed that locus 14q24.3 (ESRRB) can also be connected with high caries intensity in humans. Patients from different ethnic groups $(n=1,731)$ were examined for 25 SNPs of the ESRRB gene. Few SNPs were connected with caries prevalence in a Filipino population. In 2 families, 1 Turkish and the other Czech, increased caries intensity was noticed in individuals with recessive gene mutation. It was also noticed that SNP in the ESRRB gene

Gene Polymorphism in the Aetiology of Caries (rs4903419, rs6574293) leads to weakened enamel formation, which is more prone to bacterial acid dissolution, initiating the carious process.

On the basis of the literature it can be concluded that SNPs play an important role in the aetiology of caries. Some genes (AMELX, AQP5, and ESRRB) have been checked in multiple replication studies by many independent researchers and provide a solid source of information for being the causal variant of the disease. The majority of the studied SNPs were in Hardy-Weinberg equilibrium. However, several published studies included in the present review reported so-called "suggestive" loci ( $p=$ $5 e-8)$ with some level of biological plausibility for caries. Considering the number of hypothesis tested and the lack of replication studies, such suggestive signals could potentially be false positive, which could be a limitation of the review. Also, there is the issue of cases and controls in 
the presented studies, for whom there is a doubt that they were always perfectly matched. The caries-free or cariesaffected group qualification and the age of the patients should also be taken into consideration. In addition, cohort and case-control studies linking a particular gene to dental caries are too few, and these studies often neglect the role of environmental factors in dental caries development that could interact with genetic factors to induce disease. The authors emphasize that some additional studies should be performed to confirm previously obtained results in different populations, dentition types, or tooth surfaces [Zeng et al., 2013, 2014; Opal et al., 2015]. The contribution of epigenetic or environmental factors should also not be forgotten. Genetic mechanisms that modulate tooth tissue development, immune response, saliva functions, or carbohydrate metabolism are influenced by diet, hygiene, and other environmental factors. Although they all coexist in caries aetiology, the research studies usually focus on one of these components. Only a small number of studies was dedicated to analysing the interactions of genetic and environmental factors [Wang et al., 2012b; Chaussain et al., 2014; Abbasoğlu et al., 2015; Shaffer et al., 2015; Yildiz et al., 2016]. Future studies should associate some further information, such as metagenomics, protein-protein interaction, and gene ex- pression, as they may be linked with caries [Vieira et al., 2014; Nibali et al., 2016]. Meticulous identification of every risk factor and exactly defining their mutual relations can lead to a better understanding of the character of caries in patients and hence, better prevention.

\section{Disclosure Statement}

The authors have no conflicts of interest and no financial relationships relevant to this paper to disclose.

\section{Author Contributions}

P. Piekoszewska-Ziętek conceptualized and designed the study, collected and synthesized the data, drafted the manuscript, reviewed the manuscript for important intellectual content, and approved the final manuscript as submitted. Dr. A. Turska-Szybka conceptualized the study, supervised data collection, drafted the manuscript, critically reviewed and revised the manuscript for important intellectual content, and approved the final manuscript as submitted. Prof. D. Olczak-Kowalczyk conceptualized and designed the study, coordinated and supervised data collection, drafted the manuscript, critically reviewed and revised the manuscript for important intellectual content, and approved the final manuscript as submitted.

\section{References}

Abbasoğlu Z, Tanboğa İ, Küchler EC, Deeley K, Weber M, Kaspar C, Korachi M, Vieira AR: Early childhood caries is associated with genetic variants in enamel formation and immune response genes. Caries Res 2015;49: $70-77$.

Anjomshoaa I, Briseño-Ruiz J, Deeley K, Poletta FA, Mereb JC, Leite AL, Barreta PA, Silva TL, Dizak P, Ruff T, Patir A, Koruyucu M, Abbasoğlu Z, Casado PL, Brown A, Zaky SH, Bayram M, Küchler EC, Cooper ME, Liu K, Marazita ML, Tanboğa İ, Granjeiro JM, Seymen F, Castilla EE, Orioli IM, Sfeir C, Owyang $\mathrm{H}$, Buzalaf MA, Vieira AR: Aquaporin 5 interacts with fluoride and possibly protects against caries. PLoS One 2015;10:e143068.

Azevedo LF, Pecharki GD, Brancher JA, Cordeiro CA Jr, Medeiros KG, Antunes AA, Arruda ES, Werneck RI, de Azevedo LR, Mazur RF, Moysés SJ, Moysés ST, Faucz FR, Trevilatto PC: Analysis of the association between lactotransferrin (LTF) gene polymorphism and dental caries. J Appl Oral Sci 2010;18:166170.

Brancher JA, Pecharki GD, Doetzer AD, Medeiros KG dos S, Cordeiro Junior CA, Sotomaior VS, Bauer P, Trevilatto PC: Analysis of polymorphisms in the lactotransferrin gene promoter and dental caries. Int J Dent 2011; 2011:571726.

Buczkowska-Radlińska J, Pol J, Szmidt M, Bińczak-Kuleta A: The influence of polymorphism of the MUC7 gene on the teeth and dental hygiene of students at a faculty of dentistry in Poland. Postepy Hig Med Dosw (Online) 2012;66:204-209.

Chaussain C, Bouazza N, Gasse B, Laffont AG, Opsahl Vital S, Davit-Béal T, Moulis E, Chabadel O, Hennequin M, Courson F, Droz D, Vaysse F, Laboux O, Tassery H, Carel JC, Alcais A, Treluyer JM, Beldjord C, Sire JY: Dental caries and enamelin haplotype. J Dent Res 2014;93:360-365.

Darshana B, Veeara R, Nandita K: Influence of genetic factor on dental caries. Indian J Res Pharm Biotechnol 2014;2:1196-1207.

Deeley K, Letra A, Rose EK, Brandon CA, Resick JM, Marazita ML, Vieira AR: Possible association of amelogenin to high caries experience in a Guatemalan-Mayan population. Caries Res 2008;42:8-13.

Duverger O, Ohara T, Shaffer JR, Donahue D, Zerfas P, Dullnig A, Crecelius C, Beniash E, Marazita ML, Morasso MI: Hair keratin mu- tations in tooth enamel increase dental decay risk. J Clin Invest 2014;124:5219-5224.

Fine DH, Toruner GA, Velliyagounder K, Sampathkumar V, Godboley D, Furgang D: A lactotransferrin single nucleotide polymorphism demonstrates biological activity that can reduce susceptibility to caries. Infect Immun 2013;81:1596-1605.

Fushan AA, Simons CT, Slack JP, Manichaikul A, Drayna D: Allelic polymorphism within the TAS1R3 promoter is associated with human taste sensitivity to sucrose. Curr Biol 2009;19: 1288-1293.

Gasse B, Grabar S, Lafont AG, Quinquis L, Opsahl Vital S, Davit-Béal T, Moulis E, Chabadel O, Hennequin M, Courson F, Droz D, Vaysse F, Laboux O, Tassery H, Al-Hashimi N, Boillot A, Carel JC, Treluyer JM, Jeanpierre M, Beldjord C, Sire JY, Chaussain C: Common SNPs of AmelogeninX (AMELX) and dental caries susceptibility. J Dent Res 2013;92:418-424.

Haznedaroğlu E, Koldemir-Gündüz M, BakırCoşkun N, Bozkuş HM, Çağatay P, SüsleyiciDuman B, Mentes A: Association of sweet taste receptor gene polymorphisms with dental caries experience in school children. Caries Res 2015;49:275-281. 
Jiang Z, Wang H, Michal JJ, Zhou X, Liu B, Woods LCS, Fuchs RA: Genome wide sampling sequencing for SNP genotyping: methods, challenges and future development. Int J Biol Sci 2016;12:100-108.

Kang SW, Yoon I, Lee HW, Cho J: Association between AMELX polymorphisms and dental caries in Koreans. Oral Dis 2011;17:399-406.

Koç Öztürk L, Ulucan K, Akyüz S, Furuncuoglu $\mathrm{H}$, Bayer $\mathrm{H}$, Yarat A: The investigation of genetic polymorphisms in the carbonic anhydrase VI gene exon 2 and salivary parameters in type 2 diabetic patients and healthy adults. Mol Biol Rep 2012;39:5677-5682.

Krasone K, Lāce B, Akota I, Care R, Deeley K, Küchler EC, Vieira AR: Genetic variation in the promoter region of beta-defensin 1 (DEFB 1 ) is associated with high caries experience in children born with cleft lip and palate. Acta Odontol Scand 2014;72:235-240.

Küchler EC, Feng P, Deeley K, Fitzgerald CA, Meyer C, Gorbunov A, Bezamat M, Reis MF, Noel J, Kouzbari MZ, Granjeiro JM, Antunes LS, Antunes LA, Abreu FV, Costa MC, Tannure PN, Seymen F, Koruyucu M, Patir A, Vieira AR: Fine mapping of locus Xq25.127-2 for a low caries experience phenotype. Arch Oral Biol 2014;59:479-486.

Li ZQ, Hu XP, Zhou JY, Xie XD, Zhang JM: Genetic polymorphisms in the carbonic anhydrase VI gene and dental caries susceptibility. Genet Mol Res 2015;14:5986-5993.

Morrison J, Laurie CC, Marazita ML, Sanders AE, Offenbacher S, Salazar CR, Conomos MP, Thornton T, Jain D, Laurie CA, Kerr KF, Papanicolaou G, Taylor K, Kaste LM, Beck JD, Shaffer JR: Genome-wide association study of dental caries in the Hispanic Communities Health Study/Study of Latinos (HCHS/SOL). Hum Mol Genet 2016;25:807-816.

Nibali L, Di Iorio A, Tu YK, Vieira AR: Host genetics role in the pathogenesis of periodontal disease and caries. J Clin Periodontol 2017; 44(suppl 18):S52-S78.

Olszowski T, Adler G, Janiszewska-Olszowska J, Safranow K, Kaczmarczyk M: MBL2, MASP2, $A M E L X$, and ENAM gene polymorphisms and dental caries in Polish children. Oral Dis 2012;18:389-395.

Opal S, Garg S, Jain J, Walia I: Genetic factors affecting dental caries risk. Aust Dent J 2015;60: 2-11.

Ozturk A, Famili P, Vieira AR: The antimicrobial peptide DEFB1 is associated with caries. J Dent Res 2010;89:631-636.

Patir A, Seymen F, Yildirim M, Deeley K, Cooper ME, Marazita ML, Vieira AR: Enamel formation genes are associated with high caries experience in Turkish children. Caries Res 2008; 42:394-400.

Peres RC, Camargo G, Mofatto LS, Cortelazzi KL, Santos MC, Nobre-dos-Santos M, Bergamaschi CC, Line SR: Association of polymorphisms in the carbonic anhydrase 6 gene with salivary buffer capacity, dental plaque $\mathrm{pH}$, and caries index in children aged 7-9 years. Pharmacogenomics J 2010;10:114-119.
Petersen PE: The World Oral Health Report 2003: continuous improvement of oral health in the 21 st century - the approach of the WHO Global Oral Health Programme. Community Dent Oral Epidemiol 2003;31:3-24.

Robino A, Bevilacqua L, Pirastu N, Situlin R, Di Lenarda R, Gasparini P, Navarra CO: Polymorphisms in sweet taste genes (TAS1R2 and GLUT2), sweet liking, and dental caries prevalence in an adult Italian population. Genes Nutr 2015;10:485.

Shaffer JR, Carlson JC, Stanley BO, Feingold E, Cooper M, Vanyukov MM, Maher BS, Slayton RL, Willing MC, Reis SE, McNeil DW, Crout RJ, Weyant RJ, Levy SM, Vieira AR, Marazita ML: Effects of enamel matrix genes on dental caries are moderated by fluoride exposures. Hum Genet 2015;134:159167.

Shaffer JR, Feingold E, Wang X, Lee M, Cuenco KT, Weeks DE, Weyant RJ, Crout R, McNeil DW, Marazita ML: GWAS of dental caries patterns in the permanent dentition. J Dent Res 2013;92:38-44.

Shaffer JR, Wang X, Feingold E, Lee M, Begum F, Weeks DE, Cuenco KT, Barmada MM, Wendell SK, Crosslin DR, Laurie CC, Doheny KF, Pugh EW, Zhang Q, Feenstra B, Geller F, Boyd HA, Zhang H, Melbye M, Murray JC, Weyant RJ, Crout R, McNeil DW, Levy SM, Slayton RL, Willing MC, Broffitt B, Vieira AR, Marazita ML: Genome-wide association scan for childhood caries implicates novel genes. J Dent Res 2011;90:1457-1462.

Shimizu T, Ho B, Deeley K, Briseño-Ruiz J, Faraco IM Jr, Schupack BI, Brancher JA, Pecharki GD, Küchler EC, Tannure PN, Lips A, Vieira TC, Patir A, Yildirim M, Poletta FA, Mereb JC, Resick JM, Brandon CA, Orioli IM, Castilla EE, Marazita ML, Seymen F, Costa MC, Granjeiro JM, Trevilatto PC, Vieira AR: Enamel formation genes influence enamel microhardness before and after cariogenic challenge. PLoS One 2012; 7:e45022.

Shimizu T, Ho B, Deeley K, Faraco IM Jr, Poletta FA, Brancher JA, Pecharki GD, Küchler EC, Tannure PN, Lips A, Vieira TC, Patir A, Yildirim M, Mereb JC, Resick JM, Brandon CA, Cooper ME, Seymen F, Costa MC, Granjeiro JM, Trevilatto PC, Orioli IM, Castilla EE, Marazita ML, Vieira AR: Fine-mapping of 5q12.1-13.3 unveils new genetic contributors to caries. Caries Res 2013;47:273-283.

Shuler CF: Inherited risk for susceptibility to dental caries. J Dent Educ 2001;65:1038-1045.

Slayton RL, Cooper ME, Marazita ML: Tuftelin, mutans streptococci, and dental caries susceptibility. J Dent Res 2005;84:711-714.

Tannure PN, Küchler EC, Falagan-Lotsch P, Amorim LM, Raggio Luiz R, Costa MC, Vieira AR, Granjeiro JM: MMP13 polymorphism decreases risk for dental caries. Caries Res 2012a;46:401-407.

Tannure PN, Küchler EC, Lips A, Costa Mde C, Luiz RR, Granjeiro JM, Vieira AR: Genetic variation in MMP20 contributes to higher caries experience. J Dent 2012b;40:381-386.
Vieira AR: Genetics and caries - prospects. Braz Oral Res 2012;26(suppl 1):7-9.

Vieira AR, Marazita ML, McHenry TG: Genomewide scan finds suggestive caries loci. J Dent Res 2008;87:435-439.

Vieira AR, Modesto A, Marazita ML: Caries: review of human genetics research. Caries Res 2014;48:491-506.

Wang X, Shaffer JR, Zeng Z, Begum F, Vieira AR, Noel J, Anjomshoaa I, Cuenco KT, Lee MK, Beck J, Boerwinkle E, Cornelis MC, Hu FB, Crosslin DR, Laurie CC, Nelson SC, Doheny KF, Pugh EW, Polk DE, Weyant RJ, Crout R, McNeil DW, Weeks DE, Feingold E, Marazita ML: Genome-wide association scan of dental caries in the permanent dentition. BMC Oral Health 2012a;12:57.

Wang X, Willing MC, Marazita ML, Wendell S, Warren JJ, Broffitt B, Smith B, Busch T, Lidral C, Levy SM: Genetic and environmental factors associated with dental caries in children: the Iowa Fluoride Study. Caries Res 2012b;46: 177-184.

Weber ML, Hsin HY, Kalay E, BroŽková DS, Shimizu T, Bayram M, Deeley K, Küchler EC, Forella J, Ruff TD, Trombetta VM, Sencak RC, Hummel M, Briseño-Ruiz J, Revu SK, Granjeiro JM, Antunes LS, Antunes LA, Abreu FV, Costa MC, Tannure PN, Koruyucu M, Patir A, Poletta FA, Mereb JC, Castilla EE, Orioli IM, Marazita ML, Ouyang H, Jayaraman T, Seymen F, Vieira AR: Role of estrogen related receptor beta $(E S R R B)$ in DFN35B hearing impairment and dental decay. BMC Med Genet 2014;15:81.

Wendell S, Wang X, Brown M, Cooper ME, DeSensi RS, Weyant RJ, Crout R, MCNeil DW, Marazita ML: Taste genes associated with dental caries. J Dent Res 2010;89:1198-1202.

Werneck RI, Mira MT, Trevilatto PC: A critical review: an overview of genetic influence on dental caries. Oral Dis 2010;16:613-623.

Yang J, Zhu W, Chen J, Zhang Q, Wu: Genomewide two-marker linkage disequilibrium mapping of quantitative trait loci. BMC Genet 2014;15:20.

Yarat A, Ozturk LK, Ulucan K, Akyüz S, Atala H, Isbir T: Carbonic anhydrase VI exon 2 genetic polymorphism in Turkish subjects with low caries experience (preliminary study). In Vivo 2011;25:941-944.

Yildiz G, Ermis RB, Calapoglu NS, Celik EU, Türel GY: Gene-environment interactions in the etiology of dental caries. J Dent Res 2016; 95:74-79.

Zeng Z, Shaffer JR, Feingold E, Wang X, Weeks DE, Lee M, Cuenco KT, Broffitt B, Weyant RJ, Crout R, McNeil DW, Levy SM, Marazita ML: GWAS of primary dentition pit and fissure and smooth surface caries. Caries Res 2014; 48:330-338.

Zeng Z, Shaffer JR, Wang X, Feingold E, Weeks DE, Lee M, Cuenco KT, Wendell SK, Weyant RJ, Crout R, McNeil DW, Marazita ML: Genome-wide association studies of pit-and-fissure- and smooth-surface caries in permanent dentition. J Dent Res 2013;92:432-437. 